\title{
Facial palsy after temporal lobectomy for epilepsy: illustrative cases
}

\author{
Émile Lemoine, MD, MSc, ${ }^{1}$ Sami Obaid, MD, ${ }^{1}$ Laurent Létourneau-Guillon, MD, MSc, ${ }^{2}$ and Alain Bouthillier, MD, MSc ${ }^{1}$ \\ Divisions of ${ }^{1}$ Neurosurgery and ${ }^{2}$ Radiology, University of Montreal Health Center (CHUM), Montreal, Quebec, Canada
}

BACKGROUND Facial palsy is a rare, unexpected complication of temporal lobectomy (TL) for intractable epilepsy. Even without direct manipulation, the facial nerve fibers may be at risk of injury during supratentorial surgery, including TL.

OBSERVATIONS The authors presented two cases of facial palsy after unremarkable TL. In the first case, the palsy appeared in a delayed fashion and completely resolved within weeks. In the second case, facial nerve dysfunction was observed immediately after surgery, followed by progressive recovery over 2 years. The second patient had a dehiscence of the roof of the petrous bone overlying the geniculate ganglion, which put the facial nerve at risk of bipolar coagulation thermal injury.

LESSONS Two major mechanisms could explain the loss of facial nerve function after TL: surgery-related indirect inflammation of the nerve resulting in herpesvirus reactivation and delayed dysfunction (Bell's palsy) or indirect thermal damage to the geniculate ganglion through a dehiscent petrous roof.

https://thejns.org/doi/abs/10.3171/CASE2138

KEYWORDS Bell's palsy; epilepsy surgery; temporal lobectomy; delayed facial palsy; viral reactivation

Postoperative facial palsy is a commonly observed complication of many infratemporal surgeries that involve manipulation of the facial nerve or one of its branches. ${ }^{1}$ Such cranial nerve deficit can occur in the immediate postoperative period or after an interval of normal motor function, in which case the underlying pathophysiology may be related to gradual nerve edema, microcirculatory disorders secondary to vasospasm, and viral reactivation within the geniculate ganglion. ${ }^{1-8}$ Facial palsy is an exceedingly rare side effect of epilepsy surgery, which does not typically involve manipulation of the facial nerve. ${ }^{9,10}$ We report two cases of ipsilateral facial palsy after temporal lobectomy $(\mathrm{TL})$ for intractable epilepsy.

\section{Illustrative Cases}

\section{Case 1}

A 40-year-old, right-handed woman presented with a history of medically intractable seizures that manifested as déjà-vus followed by altered mental status. Magnetic resonance imaging (MRI) revealed hippocampal sclerosis (Fig. 1). Findings on electroencephalography and single photon emission computed tomography (CT) were compatible with medial temporal lobe epilepsy. She underwent an event-free anteromedial TL. No scalp block was performed before surgery. The next day, neurological examination indicated completely normal results, and postoperative imaging, including CT and MRI, revealed no complications. The patient had an uneventful hospital stay and was discharged on postoperative day 7 .

One week later, the patient presented to the emergency department with a progressive hemifacial paresis that had started 1 day earlier. Physical examination revealed a peripheral House-Brackmann (HB) grade III facial paresis that manifested on the same side as the TL. ${ }^{11}$ A diagnosis of Bell's palsy was made, and the patient was started on a course of systemic corticosteroids and antiviral therapy. Facial nerve MRI revealed contrast enhancement of the facial nerve along its trajectory within the right internal auditory canal and mastoid (Fig. 2). Two months after surgery, the patient had fully recovered her facial nerve function. She remained seizure free 2 years after surgery.

\section{Case 2}

A 34-year-old, right-handed man presented with a 17-year history of focal epilepsy related to a $2.5-\mathrm{cm}$ multiloculated cystic

ABBREVIATIONS CT = computed tomography; GSPN = greater superficial petrosal nerve; HB = House-Brackmann; MRI = magnetic resonance imaging; $\mathrm{TL}=$ temporal lobectomy. 


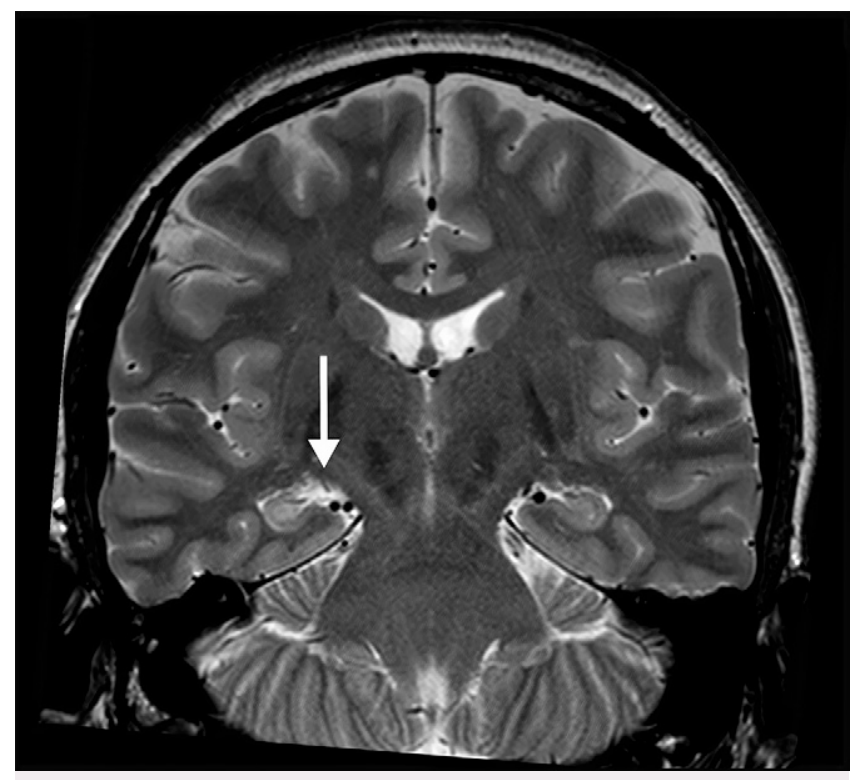

FIG. 1. Preoperative coronal T2-weighted MRI of the first patient revealed right-sided mesial temporal sclerosis (arrow).

lesion centered in the right uncus that was highly suggestive of a dysembryoplastic neuroepithelial tumor. Seizures consisted of an aura of dysosmia followed by obnubilation and orobuccal automatisms. Electroencephalographic studies confirmed the right temporal lobe origin of the seizures (Fig. 3). At that time, imaging of the facial nerve did not reveal any signal abnormality. Despite optimal

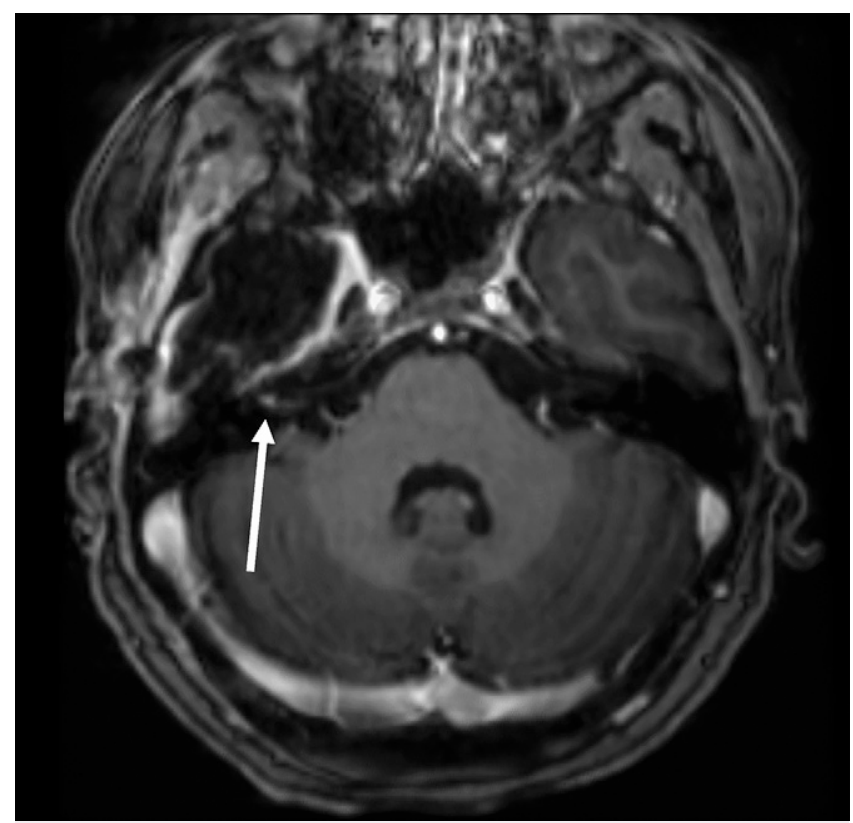

FIG. 2. Postoperative axial T1-weighted MRI of the first patient after gadolinium administration revealed abnormal enhancement of the fundus of the right internal auditory canal (arrow).

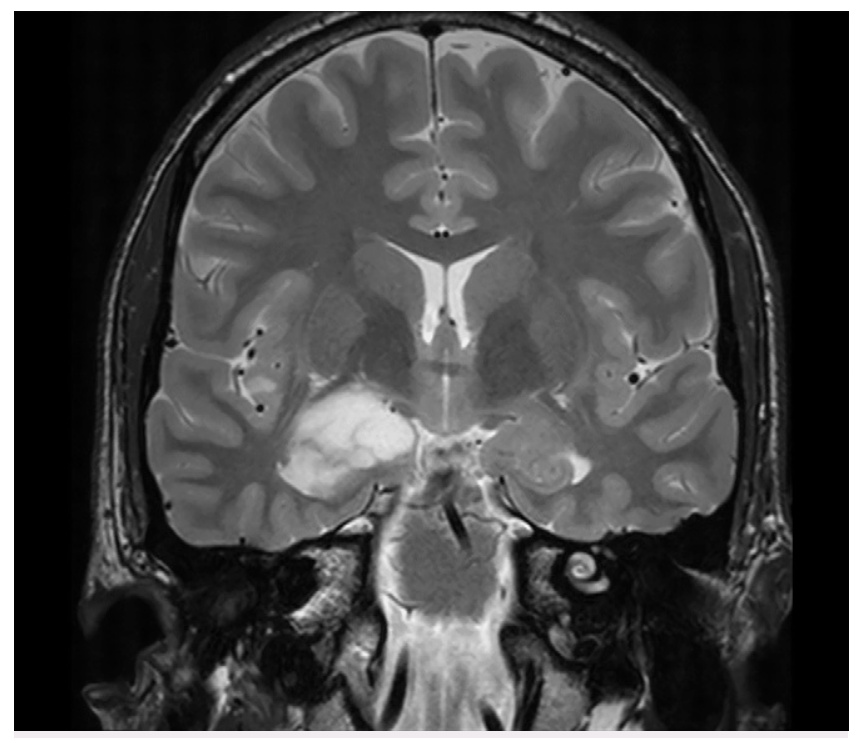

FIG. 3. Preoperative coronal T2-weighted MRI of the second patient showed a right multiloculated mesiotemporal tumor.

medical treatment, the patient's seizures became progressively more frequent.

The patient eventually underwent a right anteromedial TL that included resection of the inferior two-thirds of the tumor. A superficial bilateral scalp block was performed before the surgery, with no reported complication. Immediately after the surgery, significant facial asymmetry was noted. The next day, a right-sided HB grade III facial nerve paresis was observed. On MRI, there was no abnormal contrast enhancement of the right facial nerve. Oral corticosteroids were initiated. The facial paresis worsened to an HB grade IV over the following days. For that reason, finecut mastoid CT scanning was performed. It revealed dehiscence of the roof of the petrous bone overlying the geniculate ganglion, which put the patient at an increased risk for a traumatic facial nerve injury (Fig. 4). As a precaution, antiviral therapy was added to corticosteroid therapy.

On postoperative day 7 , the patient complained of a rightsided otalgia with new-onset vertigo and nausea. The caloric stimulation test and electronystagmography showed symmetrical vestibular function. Facial nerve MRI revealed diffuse, abnormal contrast enhancement of the right facial nerve along the meatal, labyrinthic, tympanic, mastoid, and parotid segments (Fig. 5). Because of the persistence of lagophthalmos, the patient underwent a temporary tarsorrhaphy on postoperative day 15. Eight months postoperatively, he maintained an HB grade III facial palsy. At the 18-month follow-up visit, his facial motor function had significantly improved (HB grade II) and he remained seizure free. MRI performed at that time revealed no abnormal contrast enhancement.

Of note, a few days after the patient's surgery, his pregnant wife (33 weeks' gestation) presented to the emergency department with a hemifacial weakness. She was diagnosed with an HB grade III Bell's palsy, and a course of antiviral therapy and corticosteroids was prescribed. She had fully recovered by her 2-month follow-up visit. 

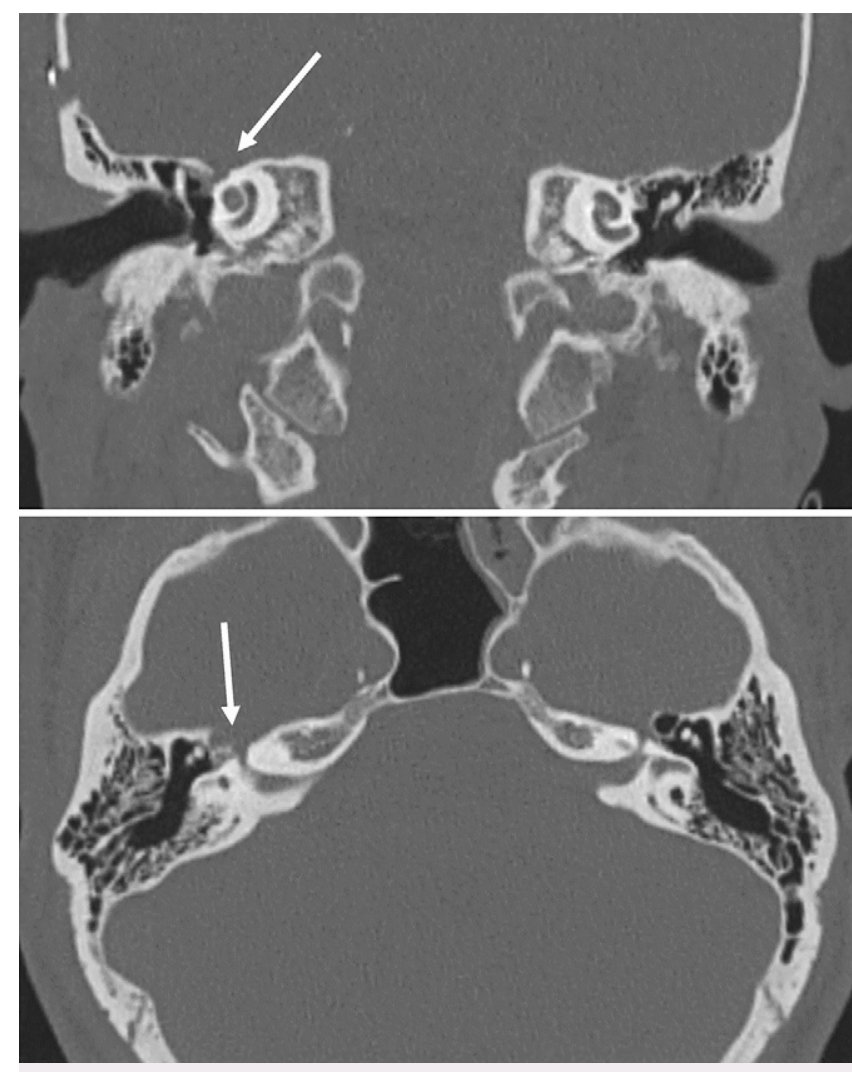

FIG. 4. Coronal (top) and axial (bottom) high-resolution temporal bone CT scans of the second patient. Bony dehiscence (arrows) was visualized in the roof of the petrous bone overlying the geniculate ganglion adjacent to the facial hiatus.

\section{Discussion \\ Observations}

Facial palsy after surgeries that involve manipulation of the facial nerve, such as infratemporal and posterior fossa surgeries, is an unsurprising complication. ${ }^{1-8}$ However, supratentorial approaches are less likely to result in such deficit, with only five cases previously published. ${ }^{9,10}$ Anderson et al. reported four cases of facial palsy after TL for refractory epilepsy. ${ }^{9}$ Three of the four cases occurred ipsilateral to the resection site, with one case on the contralateral side. In all cases, the facial palsy developed after a delay of 7 to 14 days and resolved within 6 to 8 weeks. In the article by Goldring et al. regarding 70 patients who underwent anterior TL for epilepsy, one case of transient unilateral facial palsy was noted. ${ }^{10}$ In this study, we describe how two distinct mechanisms, although similar in symptomatology, may be the culprit behind this condition, depending on the timing of the appearance of symptoms.

In case 1, facial palsy only appeared 1 week postoperatively. Delayed facial palsy after posterior fossa surgery that involves manipulation of the facial nerve is a well-known entity, occurring in $4.8 \%$ to $41 \%$ of vestibular schwannoma resections and $2.8 \%$ to $10.4 \%$ of microvascular decompressions for hemifacial spasm. ${ }^{2,5}$ Many hypotheses have been created to explain this phenomenon, the more accepted one being that of viral reactivation within the geniculate ganglion. ${ }^{1-8}$ Idiopathic facial palsy, or Bell's palsy, has been linked to herpesvirus reactivation of latent herpes simplex virus-1, varicella-zoster virus, or human herpesvirus- 6 in the geniculate ganglion, with subsequent nerve edema and compression within its most narrow course, namely the fallopian canal. ${ }^{12,13}$ Such inflammation may result in a prodrome of headache, otalgia, and tingling of the face, with rapid loss of facial tone on the ipsilateral side. ${ }^{12}$ Surgical manipulation is a well-accepted trigger for viral reactivation. ${ }^{1}$ Some series report a marked postoperative increase in herpes simplex virus-1 and varicella-zoster virus immunoglobulin $M$ and immunoglobulin $G$ in patients who develop delayed facial palsy. ${ }^{3,14,15}$ Reactivation of herpesviruses in involved dermatomes has also been described after spinal surgeries. ${ }^{16}$

In case 1 , slight indirect manipulation of the greater superficial petrosal nerve (GSPN), despite intact dural protection, may have led to stretching and inflammation of the geniculate ganglion or other segments of the facial nerve ${ }^{1,17-19}$ and concomitant enhancement on MRI (Fig. 2). Previous studies have also shown that bipolar coagulation can lead to significant axonal and myelin damage beyond its bipolar field, potentially contributing to inflammation, as was the case with our patient. ${ }^{4}$ Ultimately, viral reactivation of a predisposed inflamed nerve may have provoked the delayed facial dysfunction. In addition, up to $70 \%$ of patients with Bell's palsy completely recover within 2 months of symptom onset, even without treatment. ${ }^{20}$ Such improvement was also observed in case 1, further supporting Bell's palsy as the most likely etiology.

In case 2, facial palsy was noticed in the immediate postoperative period. Injection of a local anesthetic agent as performed in this case theoretically may have damaged the facial nerve fibers coming out of the stylomastoid foramen., ${ }^{4,21}$ However, cranial nerve deficits after a scalp block are rare, ${ }^{22}$ and the injection in our patient was performed superficially. Alternatively, we believe that the facial nerve dysfunction resulted from indirect damage to the somatic

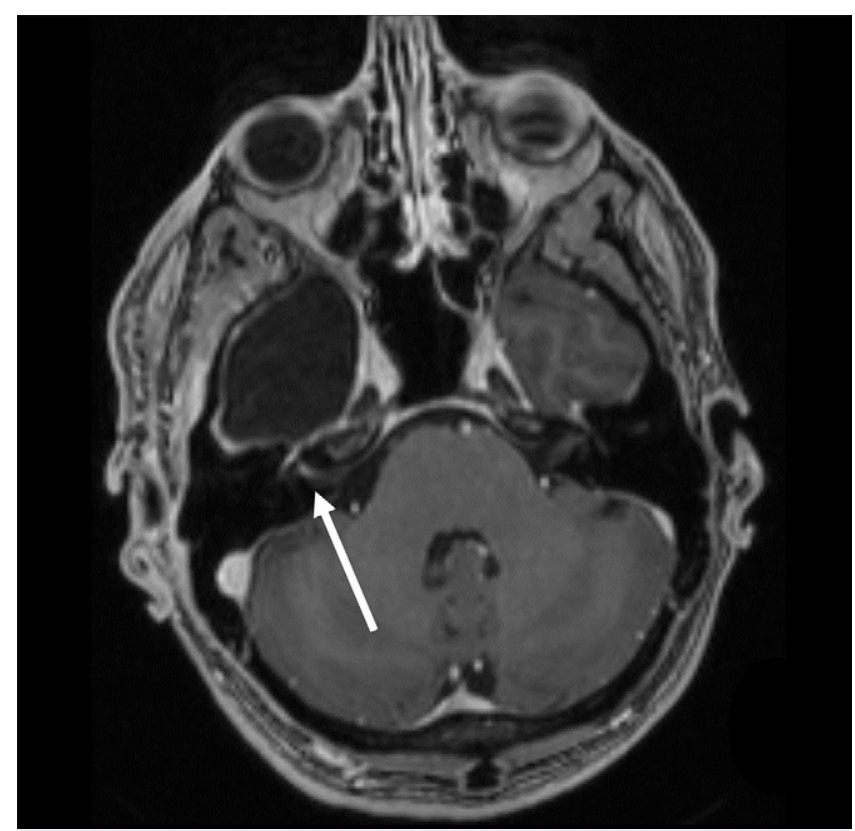

FIG. 5. Postoperative gadolinium-enhanced axial T1-weighted MRI revealed a contrast-enhancing right facial nerve within the right internal auditory canal (arrow). 
motor fibers through manipulation of the GSPN or, more plausibly, via heat transmission from bipolar coagulation. Intraoperatively, the dura remained intact; however, a thorough review of the temporal bone CT scan revealed a bony defect in the roof of the petrous bone over the facial nerve. This defect could have facilitated heat transmission and may have increased the risk of facial nerve palsy.

In a previous study on cadavers, up to $15 \%$ of patients had a bony defect in the temporal bone, exposing the geniculate ganglion under the dura. ${ }^{23}$ More recent radiological studies further corroborate such findings. ${ }^{18}$ Accordingly, indirect thermal injury to the geniculate ganglion or genu of the facial nerve in this case may have been sufficient to provoke an immediate loss of function. Recovery was also significantly delayed and incomplete after 2 years, which supported an explanation other than Bell's palsy. Preoperative imaging of the temporal bone may be useful to better assess the risk of injury to these structures and prevent injury of the exposed facial nerve. However, because of the relatively large prevalence of bony defect in the temporal bone and the rarity of postoperative facial palsy in TL, it could be reasonable to withhold imaging and assume the presence of a bony defect in all patients when performing the surgery.

In a report by Anderson et al., one of the cases occurred on the contralateral side of the resection, making iatrogenic injury of the facial nerve an improbable explanation for the observed deficit. ${ }^{9}$ Viral reactivation in dermatomes distant from the surgical site has also been reported in spinal surgeries. ${ }^{24}$ Therefore, the physical and psychological stress of undergoing surgery could, in itself, trigger reactivation of the virus. The fact that our second patient's wife presented with the same affliction is odd. Some authors have demonstrated that Bell's palsy occurs more commonly toward the end of the pregnancy; however, this is vastly debated. ${ }^{25}$ Some reports also exist of Bell's palsy clusters for which no specific etiologies have been identified. ${ }^{26-28}$ We argue that the stress associated with pregnancy, in addition to having a spouse undergoing a neurosurgical procedure, could be sufficient to trigger a viral reactivation in the patient's wife.

\section{Lessons}

Facial palsy after $T L$ is a rare and probably underreported complication. The paucity of cases reported in the literature limits the pathophysiological understanding of this entity. Although the underlying mechanism remains unproven, the proximity of the GSPN and the geniculate ganglion to the surgical site may explain why this structure is at risk of damage during TL, potentially leading to delayed viral-mediated or immediate heat-related loss of function. In addition, this study suggests that patients with a dehiscence of the roof of the petrous bone may be at increased risk of iatrogenic damage to the geniculate ganglion, which typically presents with more severe and prolonged facial nerve deficits.

\section{References}

1. Guthikonda B, Pensak ML, Theodosopoulos PV. Delayed facial palsy after the anterior petrosal approach: case report and review of the literature. Neurosurgery. 2010;66(4):E845-E846.

2. Carlstrom LP, Copeland WR III, Neff BA, et al. Incidence and risk factors of delayed facial palsy after vestibular schwannoma resection. Neurosurgery. 2016;78(2):251-255.

3. Franco-Vidal V, Nguyen DQ, Guerin J, Darrouzet V. Delayed facial paralysis after vestibular schwannoma surgery: role of herpes viruses reactivation-our experience in eight cases. Otol Neurotol. 2004;25(5):805-810.

4. Gaudin RA, Remenschneider AK, Phillips K, et al. Facial palsy after dental procedures-is viral reactivation responsible? J Craniomaxillofac Surg. 2017;45(1):71-75.

5. Lee JM, Park HR, Choi YD, et al. Delayed facial palsy after microvascular decompression for hemifacial spasm: friend or foe? J Neurosurg. 2018;129(2):299-307.

6. Révész P, Piski Z, Burián A, et al. Delayed facial paralysis following uneventful KTP laser stapedotomy: two case reports and a review of the literature. Case Rep Med. 2014;2014:971362.

7. Zalagh M, Boukhari A, Attifi $\mathrm{H}$, et al. Contralateral facial nerve palsy following mandibular second molar removal: is there co-relation or just coincidence? Pan Afr Med J. 2014;18:173.

8. Zhou Y, Song R, Li Y. Clinical characteristics and outcomes of delayed facial palsy after tympanoplasty. Acta Otolaryngol. 2015;135( 2):201-204.

9. Anderson J, Awad IA, Hahn JF. Delayed facial nerve palsy after temporal lobectomy for epilepsy: report of four cases and discussion of possible mechanisms. Neurosurgery. 1991;28(3):453-456.

10. Goldring S, Edwards I, Harding GW, Bernardo KL. Results of anterior temporal lobectomy that spares the amygdala in patients with complex partial seizures. J Neurosurg. 1992;77(2):185-193.

11. House JW, Brackmann DE. Facial nerve grading system. Otolaryngol Head Neck Surg. 1985;93(2):146-147.

12. Adour KK, Bell DN, Hilsinger RL Jr. Herpes simplex virus in idiopathic facial paralysis (Bell palsy). JAMA. 1975;233(6):527-530.

13. Schirm J, Mulkens PSJZ. Bell's palsy and herpes simplex virus. APMIS. 1997;105(11):815-823.

14. Gianoli GJ. Viral titers and delayed facial palsy after acoustic neuroma surgery. Otolaryngol Head Neck Surg. 2002;127(5):427-431.

15. Shea JJ Jr, Ge X. Delayed facial palsy after stapedectomy. Otol Neurotol. 2001;22(4):465-470.

16. Weiss R. Herpes zoster following spinal surgery. Clin Exp Dermatol. 1989;14(1):56-57.

17. Gardner WJ, Stowell A, Dutlinger R. Resection of the greater superficial petrosal nerve in the treatment of unilateral headache. $J$ Neurosurg. 1947;4(2):105-114.

18. Isaacson B, Vrabec JT. The radiographic prevalence of geniculate ganglion dehiscence in normal and congenitally thin temporal bones. Otol Neurotol. 2007;28(1):107-110.

19. Ortler M, Fiegele $T$, Walser $G$, et al. Cranial nerve monitoring during subpial dissection in temporomesial surgery. Acta Neurochir (Wien). 2011;153(6):1181-1189.

20. Jowett N, Hadlock TA. Contemporary management of Bell palsy. Facial Plast Surg. 2015;31(2):93-102.

21. Vasconcelos BC, Bessa-Nogueira RV, Maurette PE, Carneiro SC Facial nerve paralysis after impacted lower third molar surgery: a literature review and case report. Med Oral Patol Oral Cir Bucal. 2006;11(2):E175-E178.

22. Osborn I, Sebeo J. "Scalp block" during craniotomy: a classic technique revisited. J Neurosurg Anesthesiol. 2010;22(3):187-194.

23. Rhoton AL Jr, Pulec JL, Hall GM, Boyd AS Jr. Absence of bone over the geniculate ganglion. J Neurosurg. 1968;28(1):48-53.

24. Groisser D, Taylor S, Grossman ME. Postsurgical zosteriform herpes simplex 2 in noncontiguous dermatomes. J Am Acad Dermatol. 1990;23(5 pt 1):928-930.

25. Hussain A, Nduka C, Moth P, Malhotra R. Bell's facial nerve palsy in pregnancy: a clinical review. J Obstet Gynaecol. 2017;37(4):409-415.

26. Frumkin $\mathrm{H}$, Horowitz $\mathrm{M}$, Jabre JF, et al. An investigation of a workplace cluster of Bell's palsy. J Occup Med. 1992;34(11):1064-1070.

27. Reaves EJ, Ramos M, Bausch DG. Workplace cluster of Bell's palsy in Lima, Peru. BMC Res Notes. 2014;7:289.

28. Burken M, May L, Groves C, et al. Cluster of Bell's palsy cases found among hospital employees. Clin Infect Dis. 1992;15(2):381-382. 


\section{Disclosures}

The authors report no conflict of interest concerning the materials or methods used in this study or the findings specified in this paper.

\section{Author Contributions}

Conception and design: Lemoine, Obaid, Bouthillier. Acquisition of data: Lemoine, Obaid. Analysis and interpretation of data: all authors.

Drafting the article: Lemoine, Obaid, Létourneau-Guillon. Critically revising the article: Obaid, Létourneau-Guillon, Bouthillier. Reviewed submitted version of manuscript: all authors. Approved the final version of the manuscript on behalf of all authors: Lemoine. Administrative/ technical/material support: Obaid, Létourneau-Guillon, Bouthillier. Study supervision: Obaid, Bouthillier.

\section{Correspondence}

Émile Lemoine: University of Montreal Health Center (CHUM), Montreal, QC, Canada. emile.lemoine@umontreal.ca. 